\title{
Thyroid hormone response element-like sequence in anuran matrix metalloproteinase 1 gene is responsive to in vivo thyroid hormone administration
}

\author{
T Sawada $^{1}$, K Oofusa ${ }^{1}$ and K Yoshizato ${ }^{1,2,3}$ \\ ${ }^{1}$ Developmental Biology Laboratory and Yoshizato MorphoMatrix Projects (Exploratory Research for Advanced Technology, Japan Science and Technology \\ Corporation), Department of Biological Science, Graduate School of Science, Hiroshima University, 1-3-1, Kagamiyama, Higashihiroshima, \\ Hiroshima 739-8526, Japan \\ ${ }^{2}$ Laboratory for Amphibian Biology, Graduate School of Science, Hiroshima University, 1-3-1, Kagamiyama, Higashihiroshima, Hiroshima 739-8526, Japan \\ ${ }^{3}$ Hiroshima Tissue Regeneration Project, Hiroshima Prefectural Institute of Industrial Science and Technology, 3-10-32, Kagamiyama, Higashihiroshima, \\ Hiroshima 739-0046, Japan \\ (Requests for offprints should be addressed to K Yoshizato, Developmental Biology Laboratory, Department of Biological Science, Graduate School of Science, \\ Hiroshima University, 1-3-1, Kagamiyama, Higashihiroshima, Hiroshima 739-8526, Japan; Email: kyoshiz@hiroshima-u.ac.jp)
}

\begin{abstract}
Gene (Rmmp1) of matrix metalloproteinase 1 (MMP1) of the bullfrog, Rana catesbeiana, has been shown previously to contain a thyroid hormone response element (TRE)like sequence in its $5^{\prime}$-upstream region. The present study aimed to determine whether this TRE-like sequence is functional in vivo as a true TRE, and to characterize the sequences of the $5^{\prime}$-upstream region with respect to the regulation of the activity of the TRE when the TRE-like sequence was proved to be a true TRE. With this aim, various sequences of TRE-like sequence-containing $5^{\prime}$ upstream region were constructed and fused to the enhanced green fluorescent protein gene as a reporter gene. The fusion constructs were bombarded to the skin of
\end{abstract}

bullfrog tadpoles and the activity of the TRE was quantitatively determined by measuring increased intensities of fluorescence when the animals were exposed to thyroid hormone. The present study clearly demonstrated that the sequence of Rmmp 1 is a biologically active TRE in vivo. In addition, a unique $36 \mathrm{bp}$ long sequence directly flanked to the $3^{\prime}$-end of the TRE was identified which worked co-operatively with TRE to regulate the transcriptional promoter activity. It should be emphasized that the presence of TRE in the Rmmp 1 gene is unique, because its presence has not been reported in the known promoter region of vertebrate MMPs.

Journal of Endocrinology (2001) 169, 477-486

\section{Introduction}

Anuran larvae contain in their life cycle a unique postembryonic developmental process, metamorphosis, in which they extensively remodel their tissues and transform the body structure into the adult one (Yoshizato \& Frieden 1975, Niki et al. 1984, Yoshizato 1986, 1989, 1992, 1996, 1998, Oofusa \& Yoshizato 1991, Shi 2000). The remodeling consists of two processes which proceed concurrently in a tissue, the breakdown of the larval elements, and the genesis and development of the adult elements (Yoshizato 1986, 1989, 1992, Izutsu et al. 1993 , Kawai et al. 1994, Mukai et al. 1995). These two quite different processes take place under the control of the same hormone, thyroid hormone (TH). The former process has attracted researchers because it is expected that a complex but well-organized series of the regulation of the expression of genes is involved in the demolition of tissue macromolecules such as DNA and proteins. In fact, several macromolecule-degrading enzymes and their genes have been investigated in relation to the breakdown of tissues, such as cathepsin D (Weber 1967, Mukai et al. 1995), matrix metalloproteinase (MMP) 1 (Gross \& Lapiere 1962, Oofusa et al. 1994), MMP11 (Patterton et al. 1995), MMP13 (Damjanovski 1999) and MMP18 (Stolow 1996).

Recently, the understanding of anuran metamorphosis at the molecular level has been much increased in relation to the mechanism of regulation of expression of $\mathrm{TH}-$ responsive genes (Furlow \& Brown 1999, Tata 1999). Among such genes, thyroid hormone receptor (TR) gene has been best characterized in its expression during metamorphosis, in terms of promoter structures, and activators and suppressors acting on the promoter (Glass et al. 1989). It has been well established that 3,3',5'-tri-iodothyronine $\left(\mathrm{T}_{3}\right)$, an active form of $\mathrm{TH}$, acts on the $\mathrm{TH}$-responsive gene by first binding to the heterodimers of TR and retinoid X receptor (RXR) and the resulting complex of $\mathrm{T}_{3}-\mathrm{TR} / \mathrm{RXR}$ then binds to a specific sequence called thyroid hormone response element (TRE) present in the $5^{\prime}$-upstream region of the gene ( $\mathrm{Yu}$ et al. 1991). 
Gross \& Lapiere (1962) discovered collagenase (MMP1) in the tadpoles of the bullfrog, Rana catesbeiana, and this plays an essential role in the remodeling of the larval skin. We prepared specific antisera against Rana MMP1 and cloned its cDNA (Rmmp1) utilizing these antisera. The expression of MMP1 protein and Rmmp1 was greatly enhanced in bullfrog tadpoles during both $\mathrm{T}_{3}$-induced and spontaneous metamorphosis (Oofusa \& Yoshizato 1991, Oofusa et al. 1994). These studies support the notion that MMP1 is responsible for the extensive remodeling of anuran larval tissues taking place during metamorphosis (Yoshizato 1989). The sequence of the $5^{\prime}$-upstream region of Rmmp1 revealed the presence of the TRE-like sequence, $5^{\prime}$-AGGTAAGAACAGGATA-3' at -891 to - 876 (Oofusa \& Yoshizato 1996), which satisfied a general criterion for the identification of TRE proposed by Umesono et al. (1991). The binding of the complex of $T_{3}$ and TR $\beta / R X R$ to the TRE-like sequence-containing promoter of Rmmp1 gene was verified by the gel mobility shift assay and the super shift assay (Oofusa \& Yoshizato 1996). Only the region of -891 to -876 (the 'TRE'like sequence region) of Rmmp 1 in the sequence from - 1078 to +98 of Rmmp 1 was shown to be capable of binding to TR by the gel mobility shift assay. However, the biological significance of this TRE-like sequence has been uncertain for the following two reasons. Firstly, the functional in vivo assay of this specific sequence has not been done hitherto. Secondly, Rmmp1 is unique in that there are no other known genes of MMPs that contain the TRE sequence in their transcription-regulatory regions. Thus, it seems to be biologically important to determine whether this unique TRE-like sequence found in amphibia actually plays the role claimed in vivo.

These considerations led us to investigate the in vivo functions of the TRE-like sequence present in Rmmp1. With this aim, we prepared a reporter construct containing enhanced green fluorescent protein (EGFP) gene and a TRE-like sequence-containing the $5^{\prime}$-upstream region of Rmmp1. The reporter construct was transfected into tadpoles by a gold particle-mediated gene transfer system (gene gun) and the activity of the reporter gene was quantitatively determined by measuring the change in the intensity of fluorescence in the transfected tissue after treating the animals with $\mathrm{T}_{3}$. In the present study, we demonstrated that the TRE-like sequence of Rmmp 1 is biologically active in vivo and is a true TRE. In addition, we could identity a unique $36 \mathrm{bp}$ sequence in the $5^{\prime}$ upstream region which regulates TRE activity and is necessary for the $\mathrm{T}_{3}$-dependent expression of Rmmp1.

\section{Materials and Methods}

\section{Animals}

Bullfrog tadpoles were purchased from a local animal supplier and staged according to Taylor \& Kollros (1946).
The animals were raised in dechlorinated water at $24{ }^{\circ} \mathrm{C}$, and fed boiled spinach. Animals were bombarded and killed in anesthetized conditions by keeping them in iced water.

\section{Plasmid constructs}

A $5^{\prime}$-upstream region up to -1078 of Rmmp 1 was cloned as TcgM718 from a bullfrog genomic library constructed in $\lambda$ FIXII vector (Stratagene, La Jolla, CA, USA) as previously reported (Oofusa \& Yoshizato 1996). From TcgM718 a genomic DNA fragment $(-1078$ to +98$)$ was excised and subcloned into pBSKII $(+)$ (Stratagene) and used as a 'wild' fragment (Rmmp1-1078). The wild fragment contained a TRE-like sequence at -891 to -876. Eight 'mutated' fragments (Rmmp1-840, Rmmp1-524, Rmmp1-216, Rmmp1-99, Rmmp1-TRE840, Rmmp1-TRE99, Rmmp1-TREf99 and TREf) were generated from this clone (Fig. 1). Rmmp1-1078 was digested by EcoRI to remove the TRE-like sequence-containing fragment. The fragment thus obtained was Rmmp1-840 which did not contain the TRE-like sequence, but contained a sequence from -840 to +98 of the wild fragment. This Rmmp 1-840 was digested by ExoIII/MB nuclease (Sambrook et al. 1989) to obtain Rmmp1-524, Rmmp1-216, and Rmmp1-99 which also did not contain the TRE-like sequence but contained the basic common promoter sequences of AP-1 and Sp1 (Fig. 1A). Rmmp1840 and Rmmp 1-99 were ligated to the synthetic doublestranded oligonucleotides of the TRE-like sequence ( -891 to -876 ), which produced Rmmp1-TRE840 and Rmmp1-TRE99 respectively (Fig. 1B). TREf was a synthetic oligonucleotide consisting of TRE-like sequence and its $3^{\prime}$-flanking $36 \mathrm{bp}$ sequence $(-875$ to -840$)$. Rmmp1-99 was ligated to TREf to make Rmmp1TREf99. These wild and mutated fragments were inserted into pEGFP-1 (Clontech, Palo Alto, CA, USA) as shown in Fig. $1 \mathrm{~A}$ and B. As a result we obtained the following nine fragments fused to the EGFP gene: (1) pEGFP/ Rmmp1-1078, (2) pEGFP/Rmmp1-840, (3) pEGFP/ Rmmp1-524, (4) pEGFP/Rmmp1 TRE-216 and (5) pEGFP/Rmmp 1-99 as shown in Fig. 1A, and (1) pEGFP/ Rmmp1-TRE840, (2) pEGFP/Rmmp1-TRE99, (3) pEGFP/Rmmp1-TREf99 and (4) pEGFP/TREf as shown in Fig. 1B.

A plasmid containing a $5^{\prime}$-flanking region $(-1562$ to +312 ) of $x T R \beta A 1$ gene (pEGFP/TR $\beta A 1)$ (Ranjan et al. 1994) was used as a positive control construct for TH-responsive expression of the EGFP gene. Red fluorescent protein (RFP) gene was fused to the human cytomegalovirus (CMV) promoter as follows. A plasmid containing the CMV-IE94 promoter (pRc/CMV) was purchased from Invitrogen (Carlsbad, CA, USA). A BglIIHindIII fragment of $\mathrm{pRc} / \mathrm{CMV}$ containing an essential promoter region was subcloned into pDsRed1-1 (pDsRed1/CMV) which contained the open reading 
A

(1) $\mathrm{pEGFP} / R m m p 1-1078$
$(-1078$ to +98$)$

(2) $\mathrm{pEGFP} / \mathrm{Rmmp} 1-840$

$(-840$ to +98$)$

(3) pEGFP/Rmmp1-524

$(-524$ to +98$)$

(4) $\mathrm{pEGFP} / \mathrm{Rmmp} 1-216$

$(-216$ to +98$)$

(5) pEGFP/Rmmp 1-99

$(-99$ to +98$)$

pEGFP/null

(no promoter/enchancer)

B

pEGFP/Rmmp 1-1078

$(-1078$ to +98$)$

(1) pEGFP/Rmmp 1-TRE840 $(-840$ to +98 with TRE)

(2) pEGFP/Rmmp 1-TRE99 (-99 to +98 with TRE)

(3) pEGFP/Rmmp 1-TREf99 (-99 to +98 with TRE/flanking)

(4) pEGFP/Rmmp 1-TREf (TRE/flanking)

pEGFP/null

(no promoter/enchancer) $\begin{array}{lllllll}-1078 & -876 & -81 & -74 & -27 & -15 & +98\end{array}$
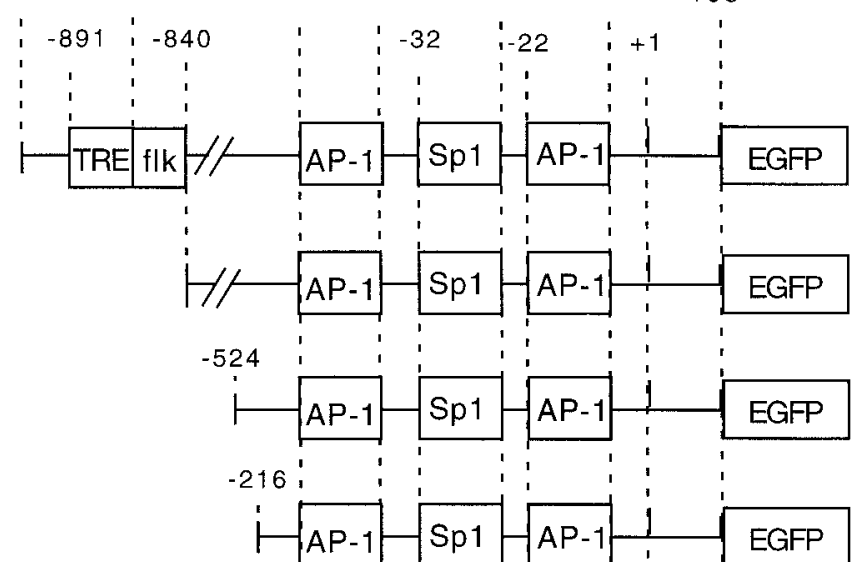

$-99: \quad 1 ; \quad: 1 ;$

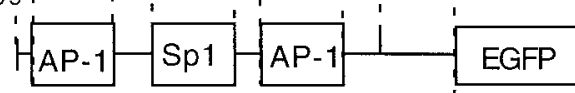

EGFP

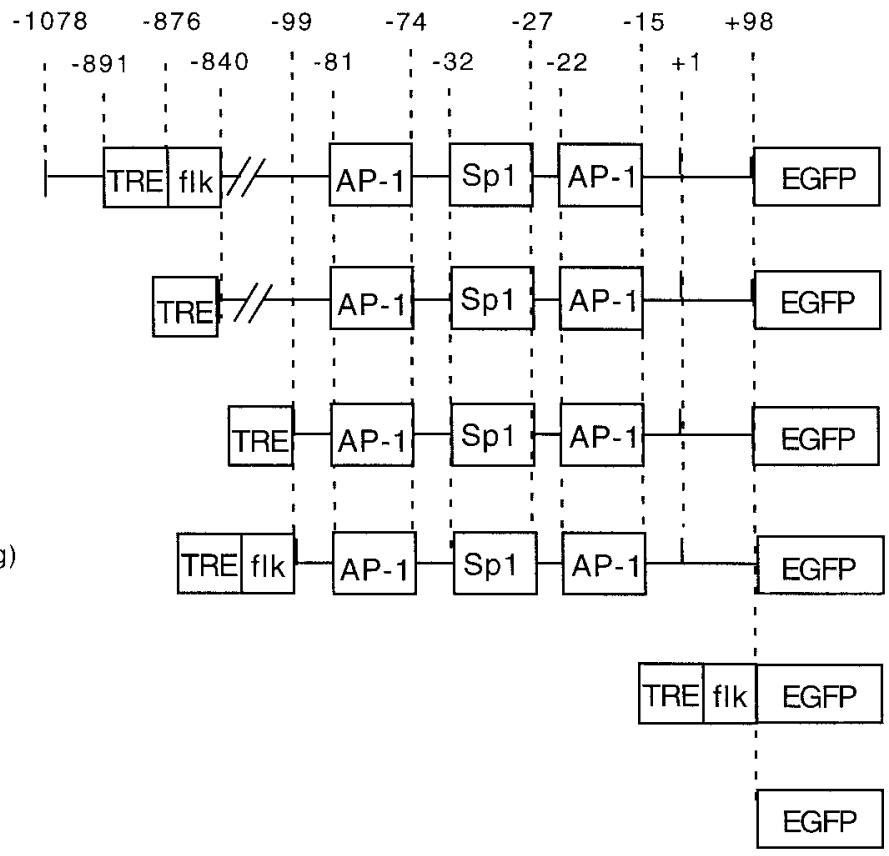

Figure 1 Schematic drawings of constructs of fusion genes. (A) Fusion genes without the TRE-like sequence. (1) Rmmp1-1078; the $5^{\prime}$-upstream region from -1078 to +98 contained a TRE-like sequence (TRE) at -891 to -876 , and potential binding sites of transcription factors, two AP-1 at -81 to -74 and -22 to -15 , and Sp1 at -32 to -27 . Four shorter constructs were made from this construct by deleting it with EcoRI and Exolll/MB nuclease: (2) Rmmp1-840 ( -840 to + 98), (3) Rmmp1-524 ( -524 to + 98), (4) Rmmp1-216 ( - 216 to +98) and (5) Rmmp1-99 ( - 99 to +98). These constructs were fused to EGFP gene producing (1) pEGFP/Rmmp1-1078, (2) pEGFP/

Rmmp 1-840, (3) pEGFP/Rmmp 1-524, (4) pEGFP/Rmmp1-216 and (5) pEGFP/Rmmp 1-99. 'flk' is the 36 bp long ( -875 to - 840 ) 3'-flanking region of the TRE-like sequence. pEGFP/null was a construct without any of these sequences of the region. (B) Deletion genes with the TRE-like sequence. Rmmp1-TRE840 (construct 1) was obtained by fusing Rmmp 1-840 to the synthetic TRE sequence. Ligation of the TRE with Rmmp 1-99 produced Rmmp1-TRE99 (construct 2). Rmmp1-TREf99 (construct 3) was obtained by ligating TREf to Rmmp1-99. TREf (construct 4) was a synthetic TRE and its 3 '-flanking region from -875 to -840 . These constructs were fused to EGFP gene producing (1) pEGFP/Rmmp1-TRE840, (2) pEGFP/Rmmp1-TRE99, (3) pEGFP/Rmmp1-TREf99 and (4) pEGFP/TREf. 
frame of RFP (Clontech). We confirmed that pDsRed1/ CMV did not respond to $T_{3}$ by the in vivo assay method described below and it was used as an internal standard to normalize the level of expression of each tested construct among experimental animals throughout this study. These plasmids were propagated by a standard protocol and purified using a Wizard Plasmid Purification System (Promega, Madison, WI, USA).

The $T_{3}$ responsiveness of the above constructs fused to the enhanced EGFP gene was quantitatively determined in vivo by utilizing a dual reporter gene system. These constructs were bombarded to tadpoles together with the construct of pDsRed1/CMV for the 'fluorescent dual reporter system'. An appropriate ratio of constructs of EGFP and RFP for a better discrimination between green (EGFP) and red (RFP) signals was found to be 3:1. Plasmid constructs $(45 \mu \mathrm{g})$ to be tested and $15 \mu \mathrm{g}$ pDsRed1/CMV as the internal control were mixed and were co-precipitated with $25 \mathrm{mg}$ spherical gold particles $(1.0 \mu \mathrm{m}$ diameter; Bio-Rad Laboratories, Hercules, CA, USA). The precipitates were suspended in $3 \mathrm{ml}$ solution of $10 \mu \mathrm{g} / \mathrm{ml}$ polyvinylpyrrolidone (Bio-Rad Laboratories) in ethanol (liquid chromatograph grade; Nacalai Tesque, Kyoto, Japan) and coated to the inner wall of a $63.5 \mathrm{~cm}$ long GoldCoat tubing (Bio-Rad Laboratories) according to the manufacturer's protocol. This tube was evenly cut into fifty $1.27 \mathrm{~cm}$ long cartridges. One piece of these cartilages containing 1.2 $\mu \mathrm{g}$ DNA was used for a bombardment to the back or tail skin of a tadpole.

\section{Gene transfer and TH treatment}

Tadpoles at stages X to XII were bombarded with the plasmids as described above at appropriate sites of the back skin or the lateral side of the tail skin using a gold particle-mediated gene transfer system (HERIOS Gene Gun; Bio-Rad Laboratories). Our preliminary tests to optimize the procedure of the gene transfer in terms of the transfer efficiency showed that the firing pressure was best at $1000 \mathrm{kPa}$ (150 pounds per square inch). We bombarded a tadpole with $0.5 \mathrm{mg}$ gold particles at each shot. A better gene transfer efficiency was obtained for the DNA loading ratio of $1.2 \mu \mathrm{g}$ plasmid DNA to $0.5 \mathrm{mg}$ gold particles for a bombardment. For the accurate estimation of the expression level of EGFP and RFP genes by photographing test animals and the following computer-aided processing of the images which are described below, it was necessary to transfer the reporter construct to the outermost layer of the epidermis as uniformly as possible. We confirmed that the above-described conditions of gene transfer using a gun satisfied this requirement. The fluorescent signal of EGFP began to be seen at $16 \mathrm{~h}$ after the bombardment whereas that of RFP was seen at $30 \mathrm{~h}$ after the bombardment. These two fluorescent signals continued to be stably expressed up to $120 \mathrm{~h}$. We measured the expression of the reporter construct of pEGFP/Rmmp 1-1078 at 0, 1, 5 and
$10 \mathrm{nM} \mathrm{T}_{3}$ (Sigma, St Louis, MO,USA). The expression increased dose-dependently up to $5 \mathrm{nM}$, where the expression reached a plateau level (data not shown). Therefore, in the present study we usually treated tadpoles at a dose of $5 \mathrm{nM}$. $\mathrm{T}_{3}$ treatment was started at $48 \mathrm{~h}$ after the bombardment when necessary.

\section{Fluorescent observations}

Animals were anesthetized in iced water for several minutes, photographed by a fluorescent dissecting microscope (MZ FLIII; Leica, Bensheim, Germany ) equipped with a pertier chilled color charge-coupled device (CCD) camera (C5810; Hamamatsu Photonics, Hamamatsu, Japan), a personal computer (PowerMacintosh G3; Apple Computer, Cupertino, CA, USA), and a high-grade printer system (EPSON, Suwa, Japan). The 'GFP' filter was used to observe the EGFP expression and the ' $G$ ' (rhodamine) filter for the RFP expression. Using this system, the ' $G$ ' filter eliminated all of the green signals. On the other hand, the 'GFP' filter could not cut off all of red signals, about $15 \%$ of RFP fluorescence contaminating the green signal. The digital camera we used for this study has three CCD chips allowing channel separation among the three primary colors. For eliminating the 'contaminated' red signals in the GFP observations, photographs were processed by using Photoshop 5.5 (Adobe System, Mountain View, CA, USA) which completely separated red signals from green signals. We were convinced that there was not a photo-bleaching problem in the fluorescence intensity of EGFP and RFP in this study from the results of the following preliminary experiments. Two tadpoles were bombarded with $\mathrm{pEGFP/CMV}$ and pDsRed/CMV respectively, and these two tadpoles were exposed to the excitation beam for $5 \mathrm{~s}$ for taking fluorescent photographs. For both constructs, such an exposure was repeated five times with $10-$ s intervals. There was no photo-bleaching in these photoimages (data not shown).

\section{Statistical analysis}

Results shown in Fig. 3 are presented as the mean \pm s.D. and their statistical analysis was performed using a paired $t$-test followed by Fisher's test to check the equivalence of the variances.

\section{Results}

We examined whether the reporter construct of TREcontaining promoter and EGFP gene can be actually expressed in vivo in response to $\mathrm{TH}$. Tadpoles at stage $\mathrm{X}$ were bombarded with the construct of $\mathrm{pEGFP/TR} \beta A 1$ as a positive control gene to demonstrate the validity of the gold particle-mediated gene transfer system as a method of gene transfer for tadpoles. The gene was shot at regions of 
$\mathrm{Oh}$

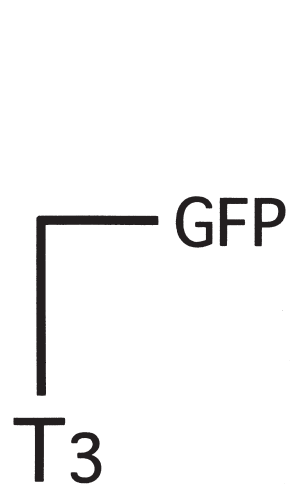

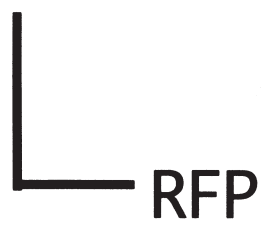
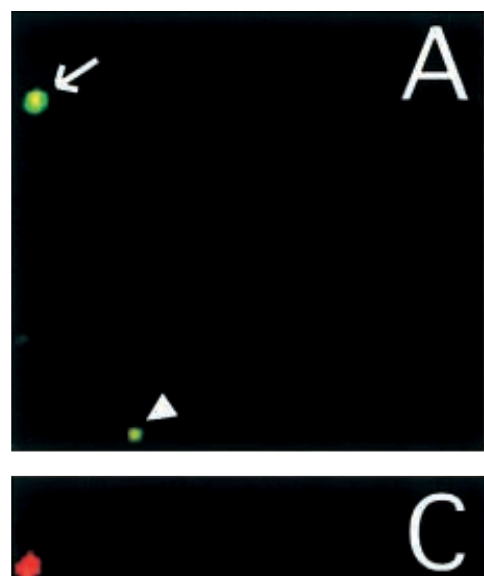

0

(1)

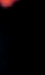

$72 \mathrm{~h}$
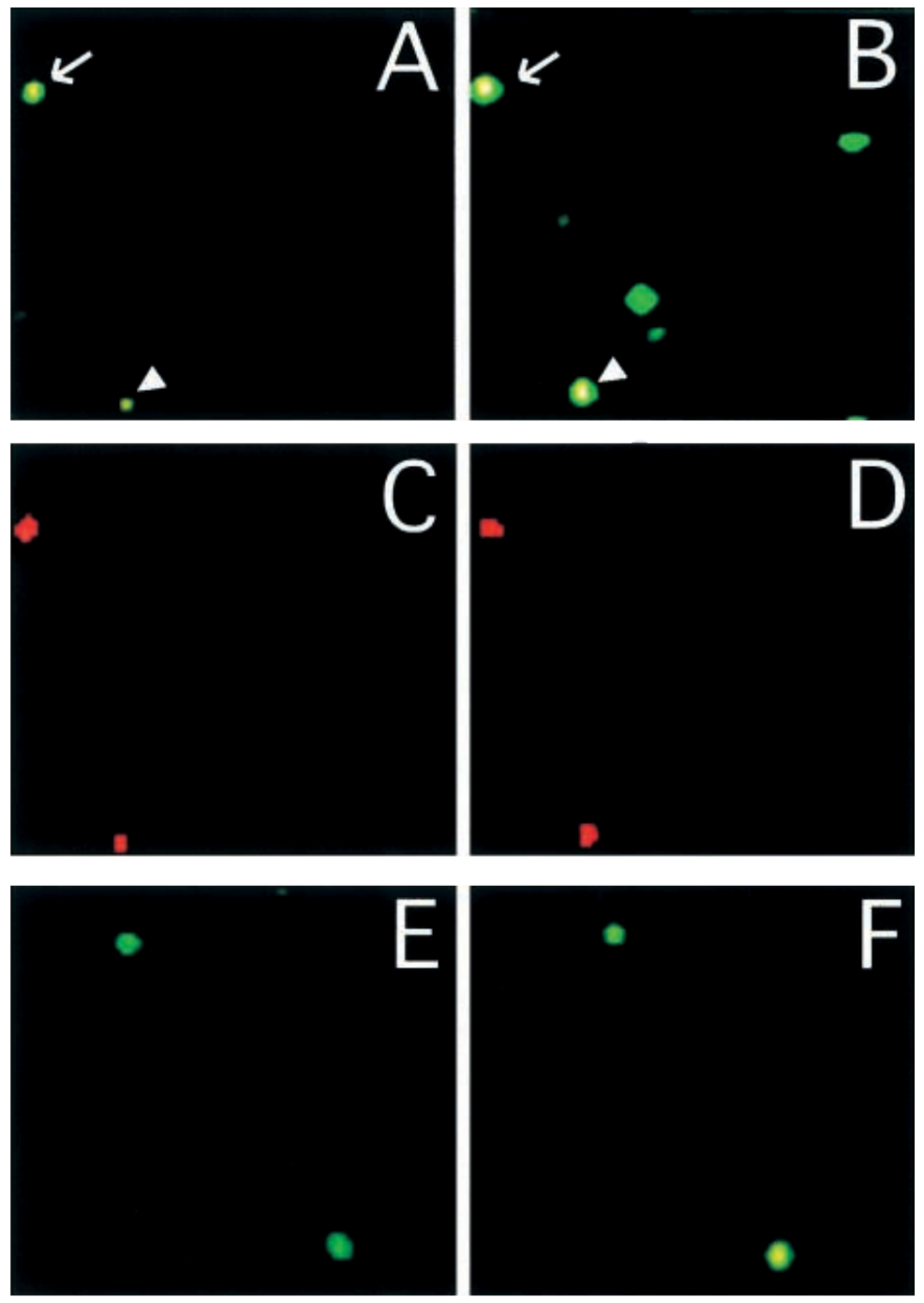

control
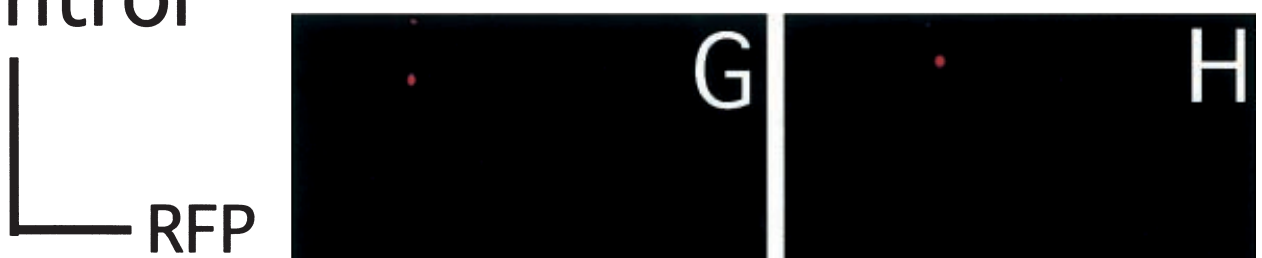

(

0

Figure 2 Responsiveness of pEGFP/Rmmp1-1078 to $T_{3}$. The mixture of two constructs, pEGFP/Rmmp 1-1078 and pDsRed1/CMV, was bombarded to the tail skin of stage $\mathrm{X}$ tadpoles. Two days later tadpoles were placed in water with $(\mathrm{A}, \mathrm{B}, \mathrm{C}, \mathrm{D})$ and without $5 \mathrm{nM} \mathrm{T}(\mathrm{E}, \mathrm{F}, \mathrm{G}, \mathrm{H})$. Tadpoles were seen through a green filter $(\mathrm{A}, \mathrm{B}, \mathrm{E}, \mathrm{F})$ and a red filter $(C, D, G, H)$ at $0 h(A, C, E, G)$ and $72 h(B, D, F, H)$ of $T_{3}$ treatment. The spots marked by the arrow and the arrowhead in $\mathrm{A}$ and $\mathrm{B}$ indicate that they are the same spot (cell) observed at the different time-period of the experiment. Bar $=0.25 \mathrm{~mm}$. 
the back skin or the lateral side of the tail skin. Two days later the animals were bathed in water with or without $5 \mathrm{nM} \mathrm{T} \mathrm{T}_{3}$. The tadpoles in $\mathrm{T}_{3}$-containing water became fluorescent at $24 \mathrm{~h}$ of the hormone treatment whereas the tadpoles in control water did not show such changes in fluorescence (data not shown). Identical responses of the fusion gene were seen at the back skin and the tail skin.

We prepared four DNA fragments from a $5^{\prime}$-upstream region of Rmmp 1 gene and made the following five constructs fused with EGFP genes: (1) pEGFP/Rmmp11078 (wild fragment), (2) $\mathrm{pEGFP} / \mathrm{Rmmp} 1-840$, (3) $\mathrm{pEGFP} /$ Rmmp 1-524, (4) pEGFP/Rmmp1-216 and (5) pEGFP/Rmmp1-99 (Fig. 1A). To measure the background level of EGFP fluorescence, pEGFP alone (pEGFP/null) was also prepared which did not contain any sequence of the $5^{\prime}$-upstream region of Rmmp 1 . Among these constructs, only pEGFP/Rmmp 1-1078 contained the TRE-like sequence. Tadpoles at stage $\mathrm{X}$ were bombarded with these constructs at the back skin and the lateral side of the tail skin, and placed in water with and without $5 \mathrm{nM} \mathrm{T}$ for $72 \mathrm{~h}$. The animals were observed through a green or red filter. The construct of pEGFP/ Rmmp1-1078 but no other constructs gave a visible enhancement of fluorescent signals when the tadpoles were exposed to $\mathrm{T}_{3}$ (Fig. 2, compare B with A). Such an enhancement was not seen in $\mathrm{T}_{3}$-free tadpoles (Fig. 2E and $F$ ). The efficiency of gene transfection appeared to be relatively constant among the tadpoles tested because red-colored spots of RFP were seen with similar frequencies among the tadpoles examined (data not shown). This situation was quantitatively demonstrated as described in Fig. 3 where the intensity of green fluorescence expressed by these constructs was calculated by computer-aided processing of photographs. $\mathrm{T}_{3}$ elevated sevenfold the fluorescence of Rmmp1-1078, but not that of other constructs (Fig. 3A). In addition, similar levels of fluorescence intensity were reproducibly obtained in these quantifications with a quite low variance for each of the tested constructs (Fig. 3), allowing us to quantitatively compare the intensity among the constructs shown in Figs 2 and 3 on a common basis. From these results we concluded that the TRE-like sequence in the 5 '-flanking region is a true TRE and is termed TRE sequence hereafter. The response of the construct of $\mathrm{pEGFP/}$ Rmmp1-1078 was quantified against the length of $\mathrm{T}_{3}$ treatment by determining the fluorescent intensities with 'Photoshop' (Fig. 4). Rmmp1-1078 responded well to $\mathrm{T}_{3}$ at $24 \mathrm{~h}$, the response continuing to increase thereafter until at least $72 \mathrm{~h}$. The increase was $6 \cdot 7$ - and 9.3-fold at $24 \mathrm{~h}$ and $72 \mathrm{~h}$ respectively. In contrast, Rmmp 1-1078 without $\mathrm{T}_{3}$ and $\mathrm{pEGFP} /$ null with and without $\mathrm{T}_{3}$ did not show any change during the experimental period.

The results of the experiments described above showed that Rmmp 1 requires the presence of the TRE sequence to respond to $\mathrm{T}_{3}$. We thought about the possibility that other sequences in the $5^{\prime}$-upstream region might regulate the activity of the TRE to respond to $T_{3}$. To test this, four additional transgenes (Rmmp1-TRE840, Rmmp1-TRE99, Rmmp1-TREf99 and TREf) were constructed by variously deleting sequences of a $3^{\prime}$-flanking region of TRE and -840 to +98 (Fig. 1B).

These constructs were fused to the EGFP gene and were given to tadpoles as above, which were then exposed to $5 \mathrm{nM} \mathrm{T} \mathrm{T}_{3}$ for $72 \mathrm{~h}$. The fluorescence intensity was quantified and is shown in Fig. 3B. Among them only the construct of Rmmp1-TREf99 significantly responded to $\mathrm{T}_{3}$, its expression being enhanced $2 \cdot 2$ times. The Rmmp 1TREf99 contained the 3 '-flanking region and sequence of -99 to +98 . When one of the two sequences was absent as in Rmmp1-TRE99 in which the former was absent or TREf in which the latter was absent, the $\mathrm{T}_{3}$ responsiveness of the TREf was lost, indicating that both regions are together required for TRE to respond to $\mathrm{T}_{3}$. The requirement of the region of -99 to +98 was understandable, because this region contained the basic promoters of AP-1 and Sp1 (Fig. 1B) which are known to be necessary for the initiation of the transcription in human gelatinase gene (Huhtala et al. 1991).

Figure 3 demonstrates that constructs of Rmmp 1-524, Rmmp1-216, Rmmp1-99 and Rmmp1-TRE840 showed a higher expression at both 0 and $72 \mathrm{~h}$ than the construct of Rmmp1-1078 at 0 h. If we define the expression of these constructs in the absence of $\mathrm{T}_{3}$ as the basic expression, the basic expression of these four constructs was significantly higher than that of constructs of Rmmp1-1078, Rmmp1840, Rmmp1-TRE99 and Rmmp1-TREf99. All these eight constructs commonly contained basic promoters of AP-1 and Sp1. The constructs of Rmmp1-1078 and Rmmp1-840 contained a sequence of -840 to -524 . Thus, this region seemed to suppress the basic expression induced by AP-1 and Sp1 to a level of the expression without AP-1 and Sp1. Comparison of the expression between Rmmp1-840 and Rmmp1-TRE840 also suggested that the TRE itself eliminates the suppressive effect of this region on the basic expression. The low or no basic expression by Rmmp1-TRE99 and Rmmp1TREf99 suggested that the TRE sequence suppresses the basic expression. Although the construct Rmmp 1-TRE840 and Rmmp1-TRE99 contained the TRE sequence, these did not respond to $T_{3}$, which again indicated the importance of the 3 -flanking sequence to potentiate the activity of the TRE sequence.

\section{Discussion}

MMP1 is suggested to be a typical housekeeping gene. Its major substrate is type I collagen which is the most abundant protein in the vertebrate body and, therefore, the enzyme is required throughout the life cycle of the tadpole to regulate the metabolism of this substrate. We have previously reported the presence of a TRE-like 


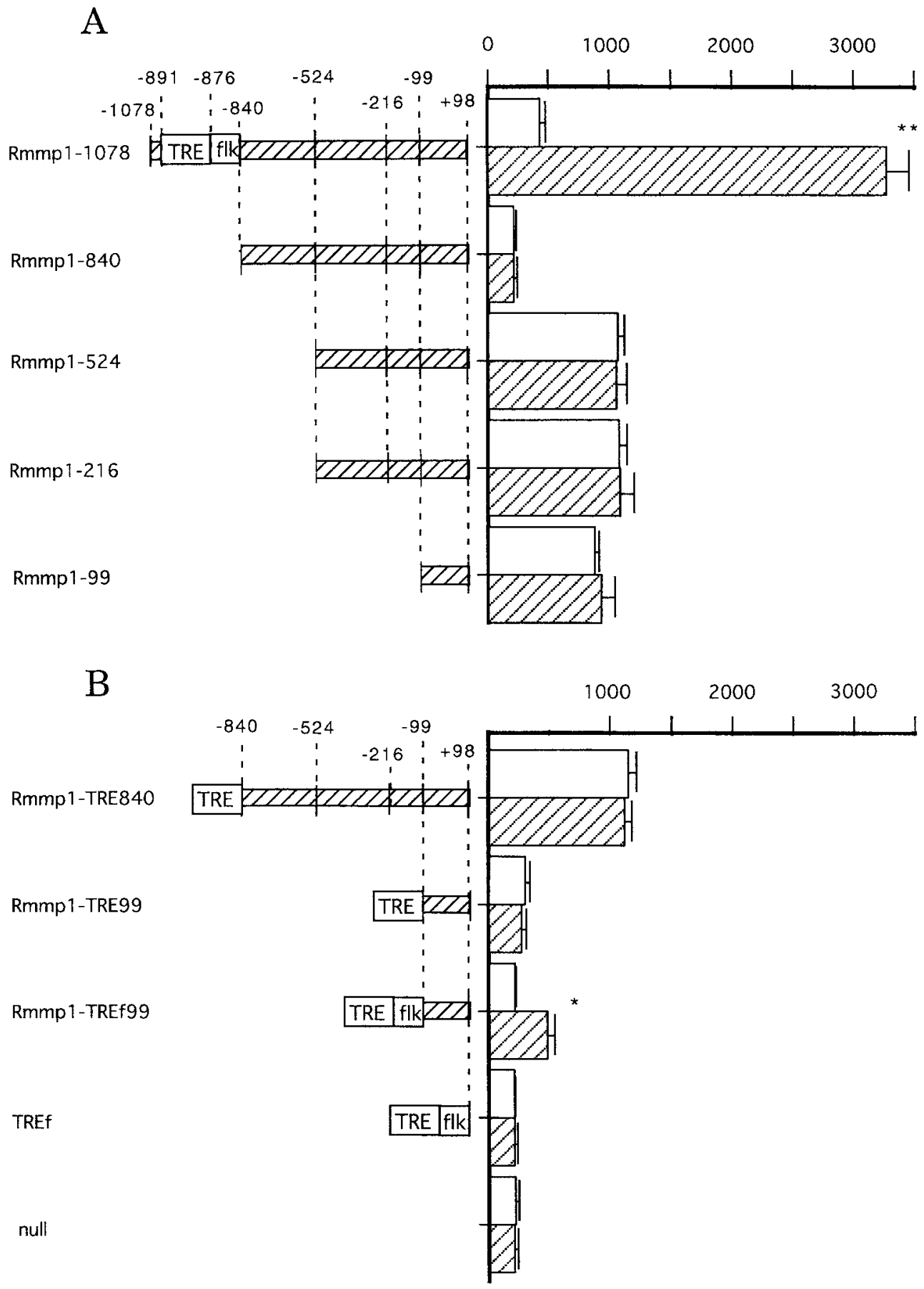

Figure 3 Quantification of the intensity of green fluorescence expressed by the transgenes. Tadpoles were transferred with ten constructs. Two days later, animals were reared for $72 \mathrm{~h}$ with (hatched bars) and without (open bars) $5 \mathrm{nM} \mathrm{T}_{3}$. The constructs of Rmmp1-1078 through Rmmp1-99 are grouped in (A) and the remaining in (B) for the sake of easy comparison. Values represent the mean of eight independent determinations (eight tadpoles) with lines showing S.E.M. ${ }^{*} P<0 \cdot 01,{ }^{*} P<0 \cdot 005$, statistically significant level between with and without $\mathrm{T}_{3}$. 


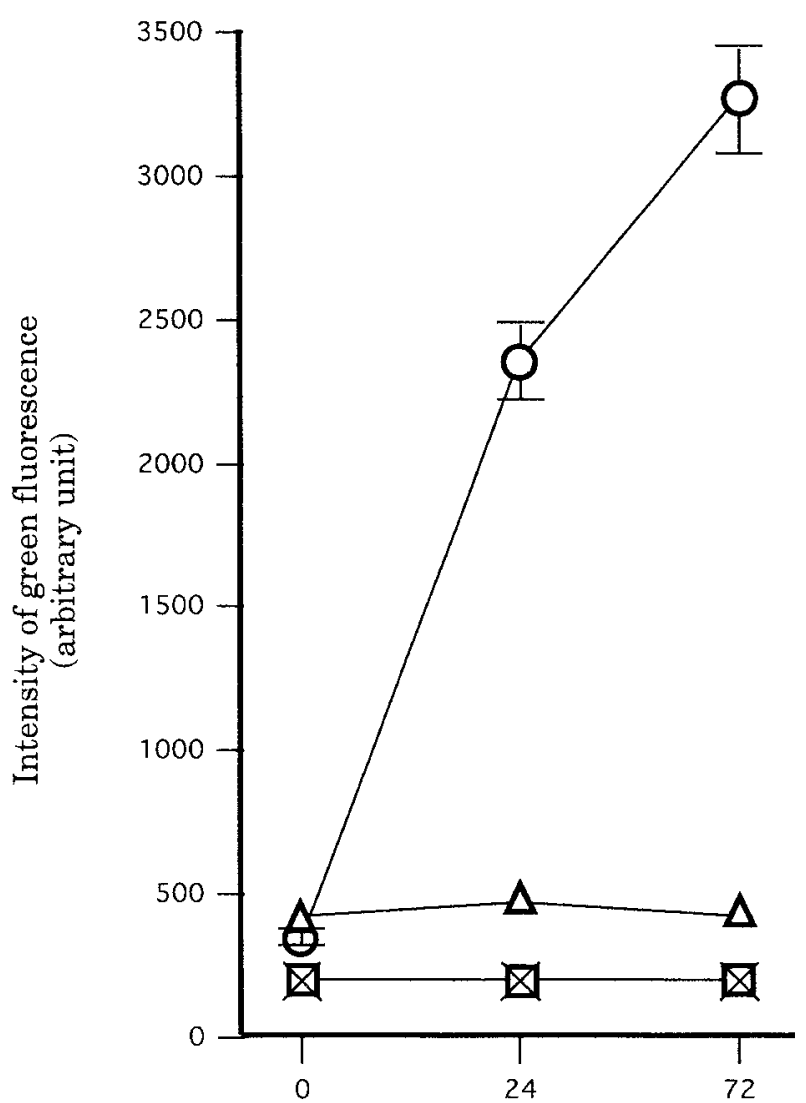

Hours with or without $\mathrm{T}_{3}$

Figure 4 Quantitation of the response of $\mathrm{pEGFP} / \mathrm{Rmmp} 1-1078$ to $\mathrm{T}_{3}$. Tadpoles were transfected with pEGFP/Rmmp1-1078 $(\bigcirc, \triangle)$ or pEGFP/null $(\square, \times)$. Two days later animals were placed in water with $(O, \square)$ and without $(\triangle, \times) 5 \mathrm{nM} \mathrm{T}_{3}$ and kept there for $72 \mathrm{~h}$. Fluorescent intensity in the gene-transferred area of the animals was quantified at 0,24 and $72 \mathrm{~h}$. The intensity of green fluorescence was normalized by dividing it by the intensity of red fluorescence. Each point represents the mean of eight determinations (eight tadpoles) with S.E.M.

sequence in the $5^{\prime}$-upstream region of Rmmp1 (Oofusa \& Yoshizato 1996). This sequence was shown to bind in vitro specifically to $\mathrm{T}_{3}$ in the presence of nuclear extracts prepared from $\mathrm{T}_{3}$-treated tadpoles. However, it remained to be determined if this TRE-like sequence is a functional TRE. This is an important question to address because, to the best of our knowledge, human MMP1 gene does not contain TRE in its promoter region and, to our knowledge, there are no other known genes of the MMP family reported hitherto which were shown to contain TRE. We asked whether such a housekeeping gene contains TRE in its transcription regulatory region. Up to now a few housekeeping genes have been reported to have a TRE sequence in their transcriptional regulation sequences, such as malic enzyme (Petty et al. 1990) and myosin heavy chain (Flink \& Morkin 1990). As shown in Fig. 1, Rmmp1 gene lacks the TATA box and has an Sp1 ( -32 to -27$)$ site adjacent to the putative transcriptional start point (Oofusa \& Yoshizato 1996). The human $92 \mathrm{kDa}$ type IV collagenase gene also lacks the TATA box in its flanking region and its Sp1 site plays an important role in the expression of the gene (Huhtala et al. 1991). The present study was performed with two aims. One was to determine if the previously reported TRE-like sequence found in Rmmp1 (Oofusa \& Yoshizato 1996) is a true TRE and functional in vivo. If the TRE-like sequence was proven to be a true TRE we had the second aim to examine whether the activity of the TRE to respond to $T_{3}$ is regulated by some specific sequences present in the $5^{\prime}$-upstream region of Rmmp 1 .

To determine the responsiveness of the TRE-like sequence-containing upstream region to $T_{3}$, we constructed a gene by fusing the upstream region to EGFP gene as a reporter gene. To find out whether EGFP is metabolically stable in vivo and can be reliably used as a reporter during an experimental period, a reporter construct consisting of a CMV promoter and an EGFP gene was constructed, and bombarded to the bullfrog tadpole skin as in the promoter of Rmmp1/EGFP gene. The expression was detectable by its fluorescence without any decay in its intensity for at least 1 week (data not shown), ensuring the usability of EGFP as a reporter in living tadpoles. In addition, we utilized a dual reporter system consisting of EGFP and RFP which can be detected through different filters. RFP reporter was used to normalize the intensity of transfected EGFP-containing genes among different tadpoles which were transfected with a different efficiency. With this dual reporter system we could correctly and reliably compare the intensity of EGFP signals among different animals. The computer software 'Photoshop' was able to separate the green signal (EGFP) and the red signal (RFP) from fluorescent signals obtained from one experimental animal, and was able to numerically measure separately each dual fluorescent intensity. To make the quantification of fluorescent signals reliable, we optimized the conditions of bombardment of the fusion genes. The distribution of the transfected cells was examined on frozen section of experimental animals. If fluorescence-positive cells were distributed differently in tissues at different levels of depth from the skin surface, we could not correctly quantify the fluorescent signals in a comparable manner among test animals, because the fluorescent intensity is reduced as the cells are at a deeper location. In fact, all fluorescent signals were found in the cells at the surface of the skin (apical cells): all of 63 positive cells among 65 frozen sections checked were in the outermost apical layer (data not shown).

We prepared nine different constructs which contained different sequences of the $5^{\prime}$-upstream region of Rmmp 1 
and fused them to the EGFP gene. These fusion genes were transferred to the back skin and the tail skin of tadpoles using a gene gun, and the fluorescent intensity was measured. The longest fragment (wild fragment, Rmmp1-1078) was shown to respond well to exogenous $5 \mathrm{nM} \mathrm{T}_{3}$ at 24 and $72 \mathrm{~h}$ after the treatment and any other tested fragments without the TRE-like sequence did not show such $\mathrm{T}_{3}$ responsiveness, clearly demonstrating that the TRE-like sequence is a true TRE.

Rmmp 1-1078 was a 5'-upstream region of Rmmp 1 with a part of the first intron covering from -1078 to +98 . The in vivo expression of this construct without $\mathrm{T}_{3}$ exposure (basic expression) was relatively low, as compared with that of Rmmp1-524, Rmmp1-216 and Rmmp1-99 which commonly lacked TRE and the sequence of -875 to -840 , but the Rmmp1-1078 was intensively upregulated when exposed to $\mathrm{T}_{3}$. These results might be explained by interpreting as follows: (1) TRElike sequence is a true TRE as concluded above, (2) TRE itself functions as a suppressor when the ligand $\left(\mathrm{T}_{3}\right)$ is not present as previously shown by Perlmann et al. (1993) and Kurokawa et al. (1993), and (3) the sequence of -875 to -840 itself or together with other sequences suppresses the basic expression. However, the last interpretation is not plausible because the basic expression of Rmmp 1-840, which lacks the sequence of -875 to -840 , was rather lower than that of the wild fragment.

The constructs of both Rmmp1-TRE840 and Rmmp1TRE99 contained the TRE, but their expression was not sensitive to $T_{3}$. Both constructs contained the sequences of -99 to +98 and, therefore, these sequences are suppressive for the function of TRE. These fragments did not commonly contain the sequence of -875 to -840 and, therefore, this $36 \mathrm{bp}$ long direct 3 '-flanking sequence was suggested to be necessary for the function of TRE, acting as its activator. This suggestion was supported by the observed expression of Rmmp1-TRE99 and Rmmp1TREf99, the latter being a fragment constructed in such a way that the $36 \mathrm{bp}$ sequence was additionally ligated to the former. This addition conferred the responsiveness to $T_{3}$ upon the Rmmp1-TRE99. In contrast to this role as an activator, the $36 \mathrm{bp}$ sequence also appears to act as a suppressor in the absence of $\mathrm{T}_{3}$, because the higher basic expression of Rmmp1-TRE840 was decreased to a level comparable with that of Rmmp1-840. Thus, it seems most likely that the region of -875 to -840 itself or part of it acts co-operatively with TRE to regulate the transcriptional promotor activity. This region did not contain any known consensus sequences which could act as a regulator of gene expression.

The present study clearly demonstrates that Rmmp 1, a typical housekeeping gene, contains a true TRE in its $5^{\prime}$-upstream promoter region. The human MMP1 gene (accession nos AF023338 and D26110) was confirmed by us not to contain either the direct repeat or the palindrome-type of TRE by surveying the TRE-like sequence on the published nucleotide sequence. There have been no reports on the structure of the genomic gene of MMP1 of other vertebrates such as birds, reptiles and fishes. The biological significance of the presence of TRE in anuran MMP1 is not clear at present. Our data support the hypothesis that a TRE in anuran MMP1 evolved to regulate the remodeling of larval tissue to adult during metamorphosis (Oofusa \& Yoshizato 1991, 1996). Further works are required to determine whether other anuran MMPs contain TRE and if this observation extends to other vertebrates besides humans.

\section{Acknowledgements}

The authors wish to thank Dr Y B Shi for his kind gift of pCAT-WT plasmid. A part of this study was supported by a Grant-in-Aid for Scientific Research (A), no. 07404057 , from the Ministry of Education, Science, Sports, and Culture of Japan.

\section{References}

Damjanovski S, Ishizuya-Oka A \& Shi YB 1999 Spatial and temporal regulation of collagenases-3, -4 , and stromelysin-3 implicates distinct functions in apoptosis and tissue remodeling during frog metamorphosis. Cell Research 9 91-105.

Flink IL \& Morkin E 1990 Interaction of thyroid hormone receptors with strong and weak cis-acting elements in the human $\alpha$-myosin heavy chain gene promoter. Journal of Biological Chemistry 265 11233-11237.

Furlow JD \& Brown DD 1999 In vitro and in vivo analysis of the regulation of a transcription factor gene by thyroid hormone during Xenopus laevis metamorphosis. Molecular Endocrinology 13 2076-2089.

Glass CK, Lipkin SM, Devary OV \& Rosenfeld MG 1989 Positive and negative regulation of gene transcription by a retinoic acid-thyroid hormone receptor heterodimer. Cell 59 697-708.

Gross J \& Lapiere CM 1962 Collagenolytic activity in amphibian tissues: a tissue culture assay. PNAS 48 1014-1022.

Huhtala P, Tuuttila A, Chow LT, Lohi J, Keski-Oja J \& Tryggvason K 1991 Complete structure of the human gene for 92-kDa type IV collagenase: Divergent regulation of expression for the 92- and 72-kilodalton enzyme genes in HT-1080 cells. Journal of Biological Chemistry 266 16485-16490.

Izutsu Y, Kaiho M \& Yoshizato K 1993 Differential distribution of epidermal basal cells in the anuran larval skin correlates with the skin's region-specific fate at metamorphosis. Journal of Experimental Zoology 267 605-615.

Kawai A, Ikeya J, Kinoshita T \& Yoshizato K 1994 A three-step mechanism of action of thyroid hormone and mesenchyme in metamorphic changes in anuran larval skin. Developmental Biology 166 477-488.

Kurokawa R, Yu VC, Naar A, Kyakumoto S, Han Z, Silverman S, Rosenfeld MG \& Glass CK 1993 Differential orientations of the DNA-binding domain and carboxy-terminal dimerization interface regulate binding site selection by nuclear receptor heterodimers. Genes and Development 7 1423-1435.

Mukai M, Obara M \& Yoshizato K 1995 Characterization of gene of anuran cathepsin D as a metamorphosis-associated enzyme. Development Growth and Differentiation 37 463-477.

Niki K, Yoshizato K, Namiki H \& Kikuyama S 1984 In vitro regression of tadpole tail by thyroid hormone. Development Growth and Differentiation 26 329-338. 
Oofusa K \& Yoshizato K 1991 Biochemical and immunological characterization of collagenase in tissues of metamorphosing bullfrog tadpoles. Development Growth and Differentiation 33 329-339.

Oofusa K \& Yoshizato K 1996 Thyroid hormone-dependent expression of bullfrog tadpole collagenase gene. Roux's Archives of Developmental Biology 205 243-251.

Oofusa K, Yomori S \& Yoshizato K 1994 Regionally and hormonally regulated expression of genes of collagen and collagenase in the anuran larval skin. International Journal of Developmental Biology 38 345-350.

Patterton D, Hayes WP \& Shi YB 1995 Transcriptional activation of the matrix metalloproteinase gene stromelysin-3 coincides with thyroid hormone-induced cell death during frog metamorphosis. Developmental Biology 167 252-262.

Perlmann T, Rangarajan PN, Umesono K \& Evans RM 1993 Determinants for selective RAR and TR recognition of direct repeat HREs. Genes and Development 7 1411-1422.

Petty KJ, Desvergne B, Mitsuhashi T \& Nikodem VM 1990 Identification of a thyroid hormone response element in the malic enzyme gene. Journal of Biological Chemistry 265 7395-7400.

Ranjan M, Wong J \& Shi YB 1994 Transcriptional repression of Xenopus TR beta gene is mediated by a thyroid hormone response element located near the start site. Journal of Biological Chemistry 269 24699-24705.

Sambrook J, Fritsch EF \& Maniatis T 1989 Molecular Cloning, edn 2. New York: Cold Spring Harbor Laboratory Press.

Shi YB 2000 Amphibian Metamorphosis. Canada: John Wiley \& Sons Inc.

Stolow MA, Bauzon DD, Li J, Sedgwick T, Liang VC, Sang QA \& Shi YB 1996 Identification and characterization of a novel collagenase in Xenopus laevis: possible roles during frog development. Molecular Biology of the Cell 7 1471-1483.

Tata JR 1999 Amphibian metamorphosis as a model for studying the development actions of thyroid hormone. Biochimie 81 359-366.
Taylor AC \& Kollros JJ 1946 Stages in the normal development of Rana pipiens larvae. Anatomical Record 94 7-23.

Umesono K, Murakami KK, Thompson CC \& Evan RM 1991 Direct repeats as selective response elements for the thyroid hormone, retinoic acid, and vitamin D3 receptors. Cell 65 1255-1266.

Weber R 1967 Biochemical and cellular aspects of tissue involution in development. Experimental Biology and Medicine 1 63-76.

Yoshizato K 1986 How do tadpoles lose their tail during metamorphosis? Zoological Science 3 219-226.

Yoshizato K 1989 Biochemistry and cell biology of amphibian metamorphosis with a special emphasis on the mechanism of removal of larval organs. International Review of Cytology 19 97-149.

Yoshizato K 1992 Death and transformation of larval cells during metamorphosis of anura. Development Growth and Differentiation 34 607-612.

Yoshizato K 1996 Cell death and histolysis in amphibian tail during metamorphosis. In Metamorphosis: Postembryonic Reprogramming of Gene Expression in Amphibian and Insect Cells, pp 647-671. Eds LI Gilbert, JR Tata \& BG Atkinson. San Diego: Academic Press.

Yoshizato K 1998 Tissue reconstitution: metamorphosis, regeneration, and artificial tissues. Wound Repair and Regeneration 6 273-275.

Yoshizato K \& Frieden E 1975 Increase in binding capacity for triiodothyronine in tadpole tail nuclei during metamorphosis. Nature 254 705-707.

Yu VC, Delsert C, Andersen B, Holloway JM, Devary OV, Naar AM, Kim SY, Boutin JM, Glass CK \& Rosenfeld MG 1991 RXR $\beta$ : a coregulator that enhances binding of retinoic acid, thyroid hormone, and vitamin $\mathrm{D}$ receptors to their cognate response element. Cell 67 1251-1266.

Received 17 January 2001

Accepted 31 January 2001 